Journal of Computer Science 4 (11): 922-927, 2008

ISSN 1549-3636

(C) 2008 Science Publications

\title{
Fuzzy Meta Node Fuzzy Metagraph and its Cluster Analysis
}

\author{
${ }^{1}$ Deepti Gaur, ${ }^{2}$ Aditya Shastri and ${ }^{1}$ Ranjit Biswas \\ ${ }^{1}$ Department of Computer Engineering, Institute of Technology and Management, \\ Sector-23/A, Gurgaon-122017, India \\ ${ }^{2}$ Department of Computer Science and Electronics, Rajasthan-304022, India
}

\begin{abstract}
Problem statement: In this study researchers propose a new fuzzy graph theoretic construct called fuzzy metagraph and a new method of clustering finding the similar fuzzy nodes in a fuzzy metagraph. Approach: We adopted T-norms (Triangular Norms) functions and join two or more Tnorms to cluster the fuzzy nodes. Fuzzy metagraph is the fuzzyfication of the crisp Metagraphs using fuzzy Generating sets and the fuzzy edge set. We could efficiently analyze the inexact information and investigate the fuzzy relation by applying the fuzzy graph theory. Results: In this study researchers suggesting a new method of clustering of a new graph theoretic structure i.e., fuzzy metagraph and investigated fuzzy metanode and fuzzy metagraph structure. Conclusion/Recommendations: Our future research will be to explore all its useful operations on fuzzy metagraph. We will give the more application based implementation of fuzzy metagraph.
\end{abstract}

Key words: Fuzzy metagraph, fuzzy metanode, cluster, fuzzy generating set

\section{INTRODUCTION}

An important concept in design of many information processing systems such as transaction processing systems, decision support systems, project management systems and workflow systems is that a graph structure. In this simplest form a graph consists of set a of elements (or nodes) and a set of ordered and unordered pairs of nodes (or edges). We would extend the fuzzy graph theory and propose a fuzzy node fuzzy graph. Since a fuzzy node fuzzy graph is complicated to analyze, we would transform it to a simple fuzzy graph by using $\mathrm{T}$-norm family $\mathrm{A}$ Graph is defined by a pair $G=\{X, E\}$ where $X=\left\{x_{1}, x_{2}, x_{3} \ldots x_{n}\right\}$ is a finite set of vertices and $\mathrm{E}$ a collection of edges that happen to connect these vertices. The edge set $\mathrm{E}$ graphically represented as $\mathrm{X} \times \mathrm{X}$. The concept of fuzzy graph is the fuzzyfication of the crisp graphs using fuzzy sets.

Definition 1: Fuzzy node fuzzy graph and T-norm: Crisp node fuzzy graph a crisp node fuzzy graph $G$ is defined by:

$$
\mathrm{G}=(\mathrm{V}, \mathrm{F}): \mathrm{V}=\left\{\mathrm{v}_{\mathrm{i}}\right\}, \mathrm{F}=\left(\mathrm{f}_{\mathrm{ij}}\right), 0 \leq \mathrm{f}_{\mathrm{ij}} \leq 1
$$

Where:

$\mathrm{V}=$ The set of the nodes

$\mathrm{F}=$ An $n \times n$ matrix whose $(i, j)$ component fij is a fuzziness of the arc from the node $v_{i}$ to the node $v_{j}$
Definition 2: Fuzzy node fuzzy graph a fuzzy node fuzzy graph $\mathrm{G}$ is defined by:

$$
\mathrm{G}=(\mathrm{V}, \mathrm{F}): \mathrm{V}=\left\{\mathrm{v}_{\mathrm{i}} / \mathrm{u}_{\mathrm{i}}\right\}, \mathrm{Y}=\left(\mathrm{y}_{\mathrm{ij}}\right), 0 \leq \mathrm{u}_{\mathrm{i}} \leq 1,0 \leq \mathrm{y}_{\mathrm{ij}} \leq 1
$$

Where:

$\mathrm{V}=$ The set of the nodes and the fuzziness

$\mathrm{ui}=\mathrm{A}$ fuzziness of the node $\mathrm{v}_{\mathrm{i}}$

$\mathrm{Y}=\mathrm{A} n \times \mathrm{n}$ matrix whose $(\mathrm{i}, \mathrm{j})$ component

$y_{i j}=A$ fuzziness of the arc from the node $v_{i}$ to the node $\mathrm{V}_{\mathrm{j}}^{[1]}$

Over the past years, a number of fuzzy graphs have been proposed to represent uncertain relationship between fuzzy elements and sets of fuzzy elements however, as mentioned before; existing fuzzy graphs are not capable of effectively modeling and directed relationships between sets of fuzzy elements.

A fuzzy node fuzzy graph is characterized by the fuzziness of the nodes and the fuzziness of the arcs. Therefore, the structure of a fuzzy node fuzzy graph is usually very complicated. Then, it should be interesting to transform a fuzzy node fuzzy graph to a crisp node fuzzy graph and we present a method to transform a fuzzy node fuzzy graph to a crisp node fuzzy graph.

\section{MATERIALS AND METHODS}

Fuzzy node fuzzy graph would be applied to the Tnorm family. As Shown in Fig. 1 every node has some

Corresponding Author: Deepti Gaur, Department of Computer Engineering, Institute of Technology and Management, Sector-23/A, Gurgaon-122017, India 
fuzzyness factor associated with it as well as every edge has some fuzzyness factor $\in[0,1]$ and this fuzzyness factor satisfies the properties of T-norm binary operations. By applying the $f_{i j}=T\left(u_{i}, y_{i j}\right)$ we get the crisp node fuzzy fuzzy edge graph.

Definition 3: Transformation from fuzzy node fuzzy graph to crisp node fuzzy graph: A fuzzy node fuzzy graph $\mathrm{G}=(\mathrm{V}, \mathrm{Y})$ can be transformed to a crisp node fuzzy graph $\mathrm{G}=(\mathrm{V}, \mathrm{F})$ by the following method. Let:

$$
\mathrm{G}=(\mathrm{V}, \mathrm{F}): \mathrm{V}=\left\{\mathrm{v}_{\mathrm{i}}\right\}, \mathrm{F}=\left(\mathrm{f}_{\mathrm{ij}}\right), \mathrm{f}_{\mathrm{ij}}=\mathrm{T}\left(\mathrm{u}_{\mathrm{i}}, \mathrm{y}_{\mathrm{ij}}\right)
$$

where, the fuzziness fij of the arc from the node $v_{i}$ to the node $\mathrm{v}_{\mathrm{j}}$ could be defined by applying $\mathrm{T}$-norms.

T -norm and its family: T-norm (Triangular Norm) is a binary operation that is defined by the following:

Definition 5: T-norm is a binary operation:

$$
\mathrm{p}, \mathrm{q} \in[0,1] \rightarrow \mathrm{T}(\mathrm{p}, \mathrm{q}) \in[0,1]
$$

Satisfying the following properties:

- $\quad$ Commutative: $\mathrm{T}(\mathrm{p}, \mathrm{q})=\mathrm{T}(\mathrm{q}, \mathrm{p})$

- Associative: $\mathrm{T}(\mathrm{p}, \mathrm{T}(\mathrm{q}, \mathrm{r}))=\mathrm{T}(\mathrm{T}(\mathrm{p}, \mathrm{q}), \mathrm{r})$

- Monotonicity: $\mathrm{p} \leq \mathrm{q}, \mathrm{r} \leq \mathrm{s} \Rightarrow \mathrm{T}(\mathrm{p}, \mathrm{r}) \leq \mathrm{T}(\mathrm{q}, \mathrm{s})$

- $\quad$ Boundary conditions: $\mathrm{T}(\mathrm{p}, 0)=0, \mathrm{~T}(\mathrm{p}, 1)=\mathrm{p}$

The typical $\mathrm{T}$-norm are following:

- $\quad$ Logical product: $\mathrm{T}_{\mathrm{L}}(\mathrm{p}, \mathrm{q})=\mathrm{p} \wedge \mathrm{q}$

- Algebraic product: $\mathrm{T}_{\mathrm{A}}(\mathrm{p}, \mathrm{q})=\mathrm{pq}$

- Multi-valued product (Lukaszewicz product): $\mathrm{T}_{\mathrm{M}}$ $(\mathrm{p}, \mathrm{q})=(\mathrm{p}+\mathrm{q}-1) \vee 0$

- $\quad$ Drastic product: $\mathrm{T}_{\mathrm{D}}(\mathrm{p}, \mathrm{q})=0$ if $\mathrm{p} \vee \mathrm{q}<1$ or $\mathrm{T}_{\mathrm{D}}(\mathrm{p}, \mathrm{q})$ $=\mathrm{p} \wedge \mathrm{q}$ if $\mathrm{p} \vee \mathrm{q}=1$

Here, $a \wedge b$ means min $(a, b), a \vee b$ means max $(a, b)$.

Definition 6: Order of t-norms: For two $\mathrm{T}$-norms $\mathrm{T}_{\mathrm{a}}$ and $\mathrm{T}_{\mathrm{b}}$ if a relation.

$\mathrm{T}_{\mathrm{a}}(\mathrm{p}, \mathrm{q}) \leq \mathrm{T}_{\mathrm{b}}(\mathrm{p}, \mathrm{q}),(\mathrm{p}, \mathrm{q}) \in[0,1]$ holds, we denote it by $\mathrm{T}_{\mathrm{a}} \leq \mathrm{T}_{\mathrm{b}}$.

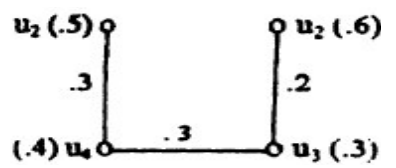

Fig. 1: G fuzzy node fuzzy edge graph $\mathrm{G}$ holds.

For any $\mathrm{T}$-norm $\mathrm{T}$, a relation $\mathrm{T}_{\mathrm{D}} \leq \mathrm{T} \leq \mathrm{T}_{\mathrm{L}}$ always

Definition 7: T -norm family: For any $\lambda \in[a, b]$, when $\mathrm{T}_{\lambda}$ is $\mathrm{T}$-norm, then we say that $\left\{\mathrm{T}_{\lambda}\right\}$ is $\mathrm{T}$-norm family that connects $\mathrm{T}_{\mathrm{a}}$ with $\mathrm{T}_{\mathrm{b}}$.

Typical T-norm families are:

- $\quad$ Dubois product: $\mathrm{T}_{\lambda}(\mathrm{p}, \mathrm{q})=\mathrm{pq} /(\mathrm{p} \vee \mathrm{q} \vee \lambda), \lambda \in[0,1]$

- Weber product: $\mathrm{T}_{\lambda}(\mathrm{p}, \mathrm{q})=0 \vee\{(1+\lambda)(\mathrm{p}+\mathrm{q}-1)-$ $\lambda \mathrm{pq}\}, \lambda \geq-1$

- $\quad$ Schweizer product: $\mathrm{T}_{\lambda}(\mathrm{p}, \mathrm{q})=\sqrt[\lambda]{\left(0 \vee \mathrm{p}^{\lambda}+\mathrm{q}^{\lambda}-1\right)}$, $\lambda>0$

- $\quad$ Quasi-logical product: $\mathrm{T}_{\lambda}(\mathrm{p}, \mathrm{q})=0$ if $\mathrm{p} \vee \mathrm{q}<1-\lambda$ or $\mathrm{T}_{\lambda}(\mathrm{p}, \mathrm{q})=\mathrm{p} \wedge \mathrm{q}$ if $\mathrm{p} \vee \mathrm{q} \geq 1-\lambda$ Here $\lambda \in[0,1]$ and so on

We could understand that by viewing the above rules that Dubois product connects logical product with algebraic product, Weber product does algebraic product with mulch-valued product, Schweizer product does multi-valued product with drastic product and quasi-logical product does logical product with drastic $\operatorname{product}^{[2-4]}$.

Fuzzy graph and cluster analysis: In order to analyze the similarity structure of nodes for a fuzzy graph, we use the symmetric relation matrix:

$$
\mathrm{S}=(\text { sij })
$$

A symmetric relation matrix $\mathrm{S}$ could be defined by using the arithmetic mean, the geometric mean, the harmonic mean and so on. Here, we define the symmetric relation matrix $S$ by using the harmonic mean $^{[4]}$.

A symmetric relation matrix $\mathrm{S}$ is defined by:

$$
\mathrm{S}=\left(\mathrm{s}_{\mathrm{ij}}\right), \frac{2}{\mathrm{~s}_{\mathrm{ij}}}=\frac{1}{\mathrm{f}_{\mathrm{ij}}}+\frac{1}{\mathrm{f}_{\mathrm{ji}}}
$$

where, $\mathrm{s}_{\mathrm{ij}}=0$ if $\mathrm{f}_{\mathrm{ij}} \bullet \mathrm{f}_{\mathrm{ij}}=0$.

In order to analyze the clustering structure among nodes, we have its max-min transitive closure:

$$
\hat{S}=\left(\hat{S}_{\mathrm{ij}}\right) \text { which is computed by } \hat{\mathrm{S}}=\mathrm{S}^{\mathrm{n}}
$$

After that we define the c-cut circuit matrix $S_{c}$ of $\hat{\mathrm{S}}=\left(\hat{\mathrm{s}}_{\mathrm{ij}}\right)$ as follows: 


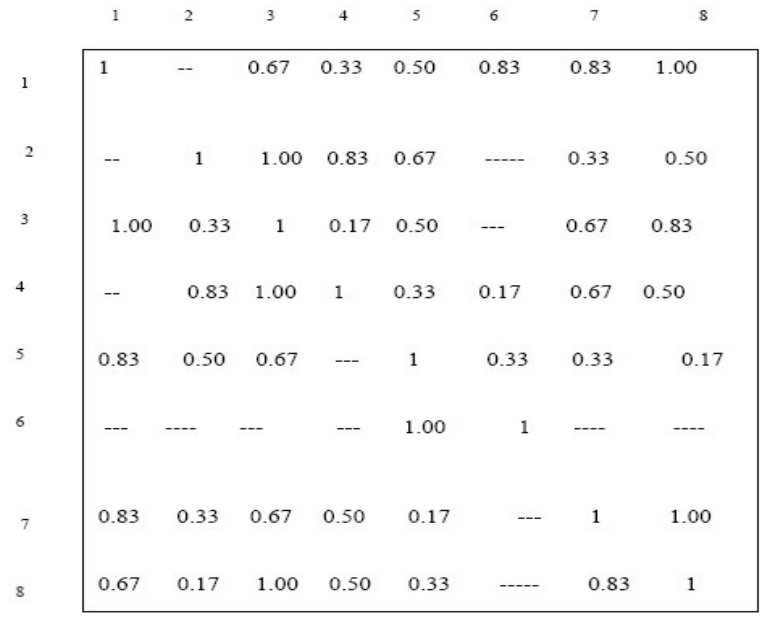

Fig. 2: Fuzzy matrix F of fuzzy graph

$\mathrm{S}_{\mathrm{c}}=\left(\mathrm{s}_{\mathrm{ij}}^{\mathrm{c}}\right)$

$$
s_{i j}^{c}=\left\{\begin{array}{l}
1 \text { if }\left(\hat{s}_{i j} \geq c\right) \\
0 \text { if }\left(\hat{s}_{i j}<c\right)
\end{array}\right.
$$

where, $(0 \leq \mathrm{c} \leq 1)$.

From the matrix $S_{c}$ we define the cluster CLsc (i):

$$
\begin{aligned}
& J_{c}(i)=\left\{j \mid s_{i j}^{c}=1,1 \leq j \leq n\right\} \\
& \operatorname{CLsc}(i)=\left\{v_{j} \mid j \in J_{c}(i)\right\}
\end{aligned}
$$

The cluster CLsc(i) gives an equivalent relationship among nodes. Then we can construct the partition tree by changing the level c of c-cut matrix which represents the clustering situation of nodes in a fuzzy graph.

Suppose a Fuzzy Matrix of a fuzzy graph is shown in Fig. 2 then we obtain the symmetric matrix $S$ in Fig. 3 and then transitive closure in Fig. 4 Conclusion is that The transitive closure matrix shows that cluster 2 and 4 have been merged at the value $\mathrm{c}=0.83$ we say that 2 and 4 are merged at cluster level R0. 83.

Concerning the cluster analysis of the fuzzy graph by viewing the above matrix we can analyzed that node 2 and 4 are equivalent so we cluster them as a single node. Similarly the node $2,7,8$ are equivalent so we cluster them and form a single node.

Following are the limitations of fuzzy graph with conventional graphs:

- A fuzzy undirected graph can represent

\begin{tabular}{|c|c|c|c|c|c|c|c|c|}
\hline & 1 & 2 & 3 & 4 & 5 & 6 & 7 & 8 \\
\hline 1 & 1 & 0.57 & 0.83 & 0.57 & 0.62 & 0.83 & 0.83 & 0.83 \\
\hline 2 & 0.57 & 1 & 0.57 & 0.83 & 0.57 & 0.57 & 0.57 & 0.57 \\
\hline 3 & 0.83 & 0.57 & 1 & 0.57 & 0.62 & 0.62 & 0.91 & 0.91 \\
\hline 4 & 0.57 & 0.83 & 0.57 & 1 & 0.57 & 0.57 & 0.57 & 0.57 \\
\hline 5 & 0.62 & 0.57 & 0.62 & 0.57 & 1 & 1.00 & 0.62 & 0.62 \\
\hline 6 & 0.62 & 0.57 & 0.62 & 0.57 & 1.00 & 1 & 0.62 & 0.62 \\
\hline 7 & 0.83 & 0.57 & 0.91 & 0.57 & 0.62 & 0.62 & 1 & 0.91 \\
\hline & 0.83 & 0.57 & 0.91 & 0.57 & 0.62 & 0.62 & 0.91 & 1 \\
\hline
\end{tabular}
relationships existing between two Variables; it cannot provide the direction of relationships

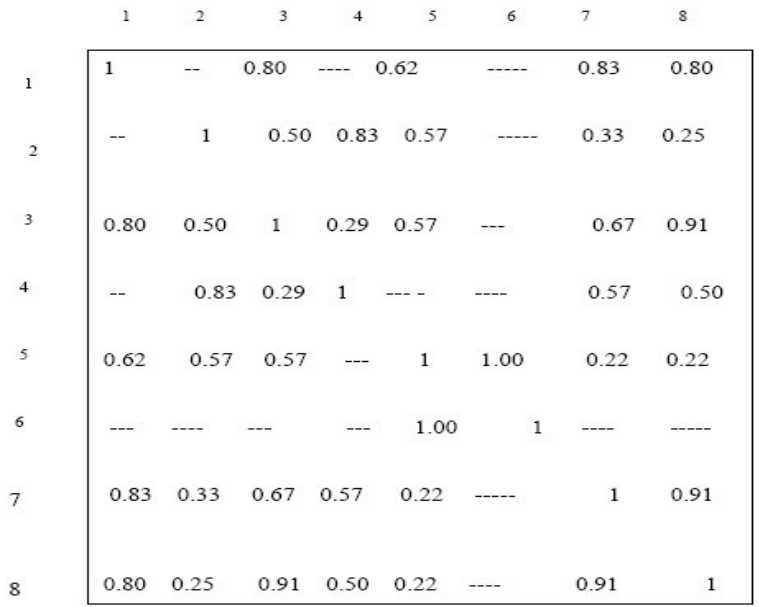

Fig. 3: Symmetric matrix $S$

Fig. 4: Transitive Closure $\hat{S}=\left(\hat{\mathrm{S}}_{\mathrm{ij}}\right)$

- The input output relationships between the element pairs can be described by the fuzzy directed graph. However, it cannot represent the relationships where there is more than one variable in the input and/or in the output

- Fuzzy hyper graph describes any fuzzy relationship as a set of fuzzy elements, but it is not able to distinguish the input variables from the outputs

- Using arcs to combine edges, a fuzzy AND/OR graph attempts to represents relationships even where there are more than one input and output variables. When describing the relationships between the sets of variables however, their are too many combined edges for fuzzy AND/OR graph to distinguish them ${ }^{[5-7]}$ 


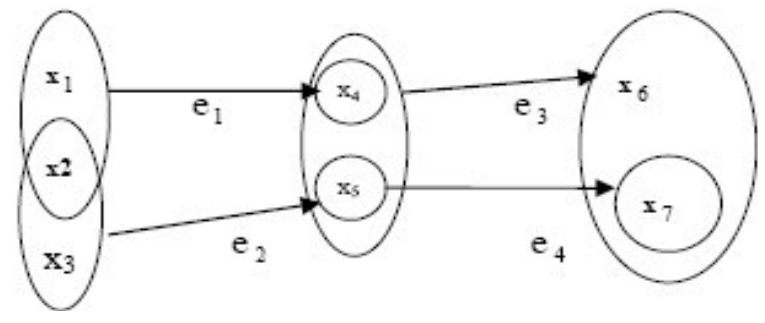

Fig. 5: Metagraph

It is critical that all the graphs lack powerful algebraic analysis methods for manipulating the directed relationships between sets of elements. This motivates the development of FM i.e., fuzzy metagraphs.

The major distinction between fuzzy metagraph and traditional graph-theoretic constructs is that an FM describes the directed relationships between sets of elements inside of single elements. Each edge in the fuzzy metagraph is an ordered pair of sets of elements. So, the edge is neither an ordered pair of elements in a fuzzy directed graph nor a disordered set of elements in a fuzzy hyper graph $^{[8-10]}$.

Metagraphs: In 1992 in the classical paper ${ }^{[12]}$, Basu and Blanning introduced the concept of metagraph. In this study we reproduce the metagraph. We begin with few definitions:

Definition: Generating set: The generating set of a metagraph is the set of elements $\mathrm{X}=\left\{\mathrm{x}_{1}, \mathrm{x}_{2} \ldots . . \mathrm{x}_{\mathrm{n}}\right\}$, which represent variables of interest.

Definition: Edge: An edge e in a metagraph is a pair $\mathrm{e}=\left\langle\mathrm{V}_{\mathrm{e}}, \mathrm{W}_{\mathrm{e}}\right\rangle \in \mathrm{E}$ (where $\mathrm{E}$ is the set of edges) consisting of an invertex $\mathrm{V}_{\mathrm{e}} \subseteq \mathrm{X}$ and an outvertex $\mathrm{W}_{\mathrm{e}} \subseteq \mathrm{X}$, each of which is a set and may contain any number of elements. The different elements in the invertex (outvertex) are co-inputs (co-outputs) of each other.

Definition: Metagraph: A metagraph $S=\langle X, E\rangle$ is a graphical representation consisting of two tuples $X$ and E. Here $X$ is its generating set and $E$ is the set of edges defined on generating sets. The generating set $\mathrm{X}$ of the metagraph $S$ i.e. the set of elements $X=\left\{x_{1}, x_{2}\right.$, $\left.\mathrm{x}_{3}, \ldots, \mathrm{x}_{\mathrm{n}}\right\}$ represents variables and occurs in the edges of the metagraphs ${ }^{[9]}$.

Example: A metagraph can be understood by the following example (Fig. 5):

$\mathrm{S}=\langle\mathrm{X}, \mathrm{E}\rangle$ is a metagraph when $\mathrm{X}=\left\{\mathrm{x}_{1}, \mathrm{x}_{2}, \mathrm{x}_{3}, \mathrm{x}_{4}, \mathrm{x}_{5}\right.$, $\left.\mathrm{x}_{6}, \mathrm{x}_{7}\right\}$ is the generating set and $\mathrm{E}=\left\{\mathrm{e}_{1}, \mathrm{e}_{2}, \mathrm{e}_{3}, \mathrm{e}_{4}\right\}$ is the set of edges.

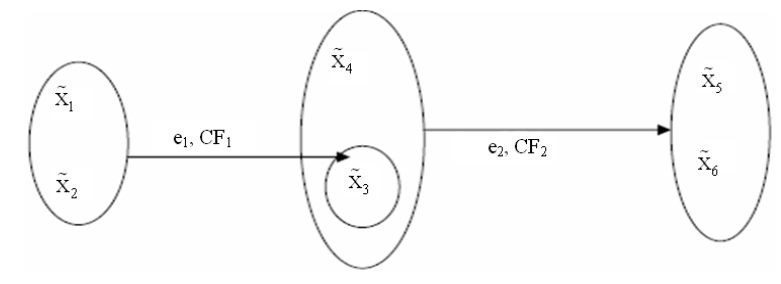

Fig. 6: Fuzzy metagraph

The edge set can be specified as:

$E=\left\{\left\langle\left\{x_{1}, x_{2}\right\},\left\{x_{4}\right\}\right\rangle,\left\langle\left\{x_{2}, x_{3}\right\},\left\{x_{5}\right\}\right\rangle,\left\langle\left\{x_{4}, x_{5}\right\},\left\{x_{6}\right.\right.\right.$, $\left.\left.\left.\mathrm{x}_{7}\right\}\right\rangle,\left\langle\left\{\mathrm{x}_{5}\right\},\left\{\mathrm{x}_{7}\right\}\right\rangle\right\}$

In-vertex is an operation (function) having one argument which can find out the internal vertices from a given set. For example:

$$
\text { In-vertex }\left(\left\langle\left\{\mathrm{x}_{4}, \mathrm{x}_{5}\right\},\left\{\mathrm{x}_{6}, \mathrm{x}_{7}\right\}\right\rangle\right)=\left\{\mathrm{x}_{4}, \mathrm{x}_{5}\right\}
$$

Out-vertex is another operation (function) having one argument which can find out what are the out vertices from the given set. For example:

$$
\text { Out-vertex }\left(\left\langle\left\{\mathrm{x}_{4}, \mathrm{x}_{5}\right\},\left\{\mathrm{x}_{6}, \mathrm{x}_{7}\right\}\right\rangle\right)=\left\{\mathrm{x}_{6}, \mathrm{x}_{7}\right\}
$$

Two more operations of metagraph are the co-input and co-output operations each having two arguments. For example:

$$
\begin{aligned}
& \text { Co-input }\left\{\mathrm{x}_{4},\left\langle\left\{\mathrm{x}_{4}, \mathrm{x}_{5}\right\},\left\{\mathrm{x}_{6}, \mathrm{x}_{7}\right\}\right\rangle\right\}=\left\{\mathrm{x}_{5}\right\} \\
& \text { Co-output }\left\{\mathrm{x}_{6},\left\langle\left\{\mathrm{x}_{4}, \mathrm{x}_{5}\right\},\left\{\mathrm{x}_{6}, \mathrm{x}_{7}\right\}\right\rangle\right\}=\left\{\mathrm{x}_{7}\right\}
\end{aligned}
$$

The edges of the metagraph are labeled as:

$$
\begin{aligned}
& \mathrm{e}_{1}=\left\langle\left\{\mathrm{x}_{1}, \mathrm{x}_{2}\right\},\left\{\mathrm{x}_{4}\right\}\right\rangle \\
& \mathrm{e}_{2}=\left\langle\left\{\mathrm{x}_{2}, \mathrm{x}_{3}\right\},\left\{\mathrm{x}_{5}\right\}\right\rangle \\
& \mathrm{e}_{3}=\left\langle\left\{\mathrm{x}_{4}, \mathrm{x}_{5}\right\},\left\{\mathrm{x}_{6}, \mathrm{x}_{7}\right\}\right\rangle \\
& \mathrm{e}_{4}=\left\langle\left\{\mathrm{x}_{5}\right\},\left\{\mathrm{x}_{7}\right\}\right\rangle
\end{aligned}
$$

Fuzzy metagraphs: The fuzzy metagraph is the concept of fuzzyfication of the crisp Metagraph using fuzzy generating set. Fuzzy generating set is the node set of all the elements of fuzzy metagraph.

Definition of fuzzy metanode fuzzy metagraph: Consider a finite set $X=\left\{x_{i},=1,2, \ldots, I\right\}$. A fuzzy metagraphs is a triplet shown in Fig. 6.

\section{RESULTS AND DISCUSSION}

Cluster in fuzzy metagraph: The Description of members of the generating sets of fuzzy metagraph of 
Fig. 7 is shown in Table 1. Fuzzy matrix as well as the Symmetric matrix of Fig. 7 is shown in Fig. 8 and 9.

$\tilde{S}=\{X, \tilde{X}, \tilde{E}\}$ where $\tilde{X}$ is a fuzzy set on $X$ is known Fuzzy MetaNode and $\tilde{\mathrm{E}}$ is a fuzzy edge set $\left\{\tilde{\mathrm{e}}_{\mathrm{k}}, \mathrm{k}=1,2,3, \ldots, \mathrm{K}\right\}$ known as fuzzy edge set.

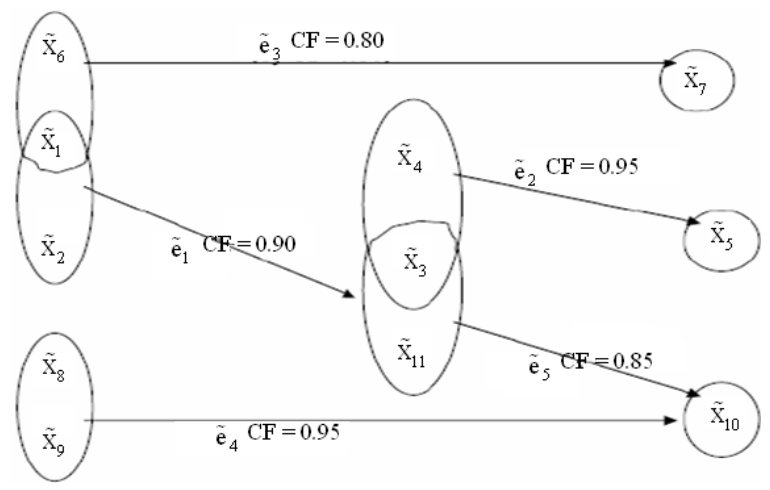

Fig. 7: A fuzzy Metagraph

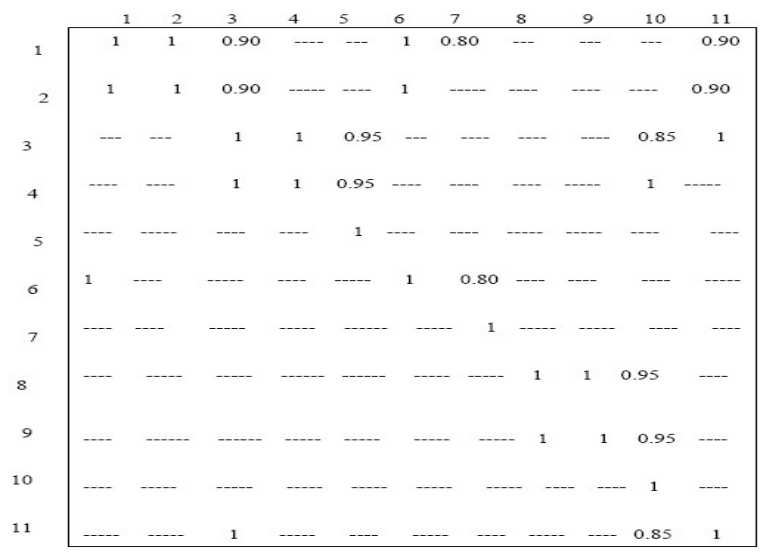

Fig. 8: Fuzzy matrix of fuzzy metagraph

Table 1: Members of generating Sets their proposition

\begin{tabular}{lll}
\hline Sr. No & Member of generating sets & Proposition \\
\hline 1 & $\tilde{\mathrm{x}}_{1}$ & Altitude is high \\
2 & $\tilde{\mathrm{x}}_{2}$ & Altitude is low \\
3 & $\tilde{\mathrm{x}}_{3}$ & control system breakdown \\
4 & $\tilde{\mathrm{x}}_{4}$ & Cabin breakdown \\
5 & $\tilde{\mathrm{x}}_{5}$ & Net breakdown \\
6 & $\tilde{\mathrm{x}}_{6}$ & System breakdown \\
7 & $\tilde{\mathrm{x}}_{7}$ & Output large \\
8 & $\tilde{\mathrm{x}}_{8}$ & Relay is abnormal \\
9 & $\tilde{\mathrm{x}}_{9}$ & Relay is normal \\
10 & $\tilde{\mathrm{x}}_{10}$ & Voltage is high \\
11 & $\tilde{\mathrm{x}}_{11}$ & Voltage is low \\
\hline
\end{tabular}

Each Component of $\tilde{\mathrm{e}}_{\mathrm{k}}$ in $\tilde{\mathrm{E}}$ is characterized by an ordered pair $\left\langle\tilde{\mathrm{V}}_{\mathrm{k}}, \tilde{\mathrm{W}}_{\mathrm{k}}\right\rangle$ in pair $\tilde{\mathrm{V}}_{\mathrm{k}} \subseteq \tilde{\mathrm{X}}$ is the invertex of $\tilde{\mathrm{e}}_{\mathrm{k}}$ and the $\tilde{\mathrm{W}}_{\mathrm{k}} \subseteq \tilde{\mathrm{X}}$ is the outvertex. The co-input of any $\tilde{\mathrm{x}} \in \tilde{\mathrm{V}}_{\mathrm{k}}$ is $\tilde{\mathrm{V}}_{\mathrm{k}}\{\tilde{\mathrm{x}}\}$ and the co-output of any $\tilde{x} \in \tilde{W}_{k}$ is $\tilde{W}_{k}\{\tilde{x}\}$.

Figure 6 fuzzy metagraph whose element set is $\mathrm{X}=\left\{\tilde{\mathrm{x}}_{1}, \ldots, \tilde{\mathrm{x}}_{6}\right\}$ is known as fuzzy MetaNode and whose edge set consists of:

$$
\tilde{\mathrm{e}}_{1}=\left\langle\left\{\tilde{\mathrm{x}}_{1}, \tilde{\mathrm{x}}_{2}\right\},\left\{\tilde{\mathrm{x}}_{3}\right\}\right\rangle \text { and } \tilde{\mathrm{e}}_{2}=\left\langle\left\{\tilde{\mathrm{x}}_{3}, \tilde{\mathrm{x}}_{4}\right\},\left\{\tilde{\mathrm{x}}_{5}, \tilde{\mathrm{x}}_{6}\right\}\right\rangle
$$

The in-vertex and out-vertex of $\tilde{\mathrm{e}}_{1}$ are $\left\{\tilde{\mathrm{x}}_{1}, \tilde{\mathrm{x}}_{2}\right\}$ and $\left\{\tilde{x}_{3}\right\}$ respectively. An important property of graph is its connectivity. In Fig. 6 there is a sequence of edges $\left\langle\tilde{e}_{1}, \tilde{\mathrm{e}}_{2}\right\rangle$ that connects $\tilde{\mathrm{x}}_{1}$ to $\tilde{\mathrm{x}}_{5}$ which means there is a path from $\tilde{\mathrm{x}}_{1}$ to $\tilde{\mathrm{x}}_{5}$ exists.

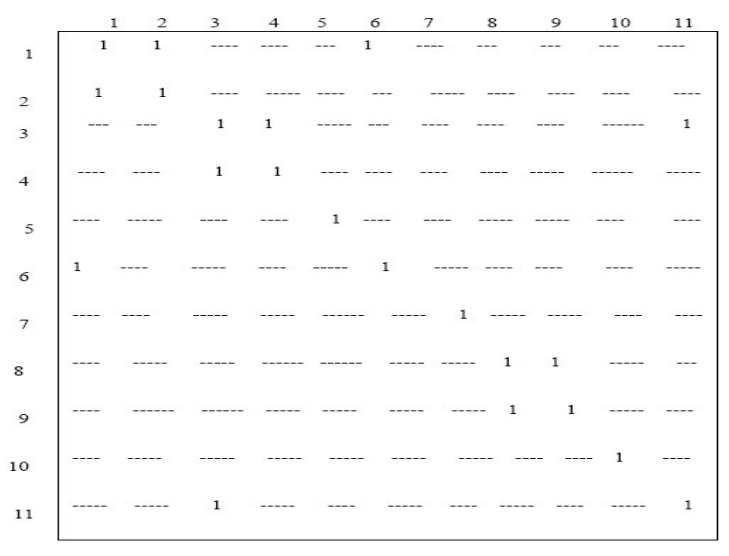

Fig. 9: Symmetric matrix $S$ of fuzzy metagraph

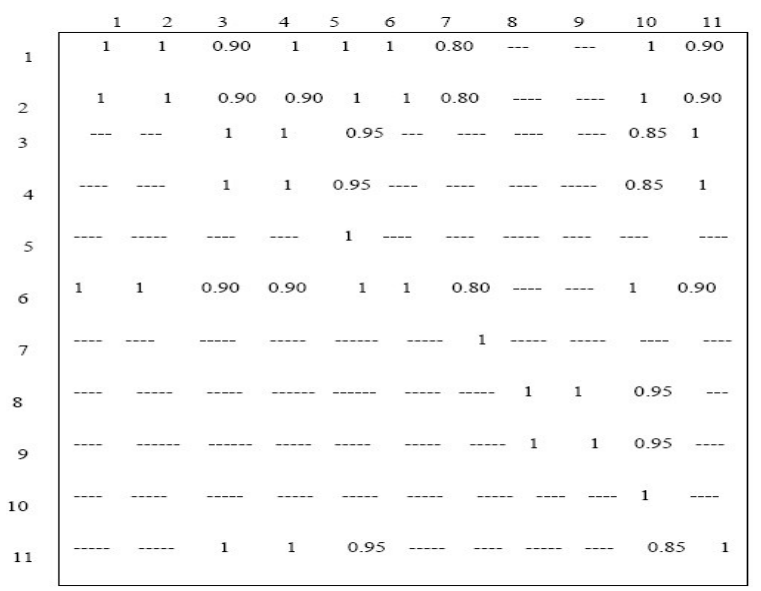

Fig. 10: Transitive closure of fuzzy metagraph $\hat{S}=\left(\hat{\mathrm{S}}_{\mathrm{ij}}\right)$ 
Fuzzy metagraphs and cluster analysis of fuzzy metagraph: The tabular representation of the Fuzzy generating set of the fuzzy Metagraph with there meaning is shown in Table 1.

In order to analyze the similarity structure of nodes for a fuzzy Metagraph, we use the symmetric relation matrix $S=$ (sij). Before that we have to construct the fuzzy matrix of Fuzzy Metagraph. Fuzzy matrix of above fuzzy Metagraph will be shown in Fig. 7.

The Symmetric matrix will be shown in Fig. 8.

Transitive closure matrix of above fuzzy matrix in Fig. 7 will be shown in Fig. 10. By viewing this transitive closure matrix we can analyze that some nodes are equivalent we will make a cluster of those nodes. In spite of putting them separately. In the above Fig. 10 node 1, 2, 6 will be clustered as a single node.

\section{CONCLUSION}

Metagraph is a graphical model that not only visualized the process of any system but also their formal analysis where the analysis will be accomplished by means of an algebraic representation of the graphical structure. The graphical structure can be represented by the adjacency and incidence matrix of a metagraph. Metagraphs have lot of applications in the field of information processing systems; decision support systems, models Management of database and rule base management of work flow systems in which the work consists of information processing tasks to be performed by the human or the machines metagraphs provide a useful and comprehensive function for modeling. In our example of metagraph the entities will correspond to the elements of the generating sets and the tasks will correspond to the edges in the metagraph. This construct extends the features offered by the traditional graph structures i.e., digraphs, hyper graphs. Metagraph allows different components of the process to be represented both graphically and analytically. Our future work will be to explore all its useful operations on fuzzy metagraph. We will give the more application based implementation of fuzzy metagraph.

\section{REFERENCES}

1. Zadeh, L.A., 1965. Fuzzy Sets*. Inform. Control, 8: 338-353.

http://www-bisc.cs.berkeley.edu/Zadeh-1965.pdf.
2. Zadesh, L.A., 1975. Fuzzy Sets and Their Application to Cognitive and Decision Processes. 1st Edn., Academic Press Inc., New York, USA., ISBN: 10: 0127752609, pp: 506.

3. Nishhida, T. and E. Takeda, 1978. Fuzzy Set and its Applications. 1st Edn., Morikita Shuppan, (In Japanese).

4. Uesu, H., 2004. Approximated analysis of fuzzy node fuzzy graph and its applications. Proceedings of the IEEE International Conference on Fuzzy Systems, July 25-29, IEEE Computer Society, Washington DC., USA., pp: 873-877. DOI: 10.1109/FUZZY.2004.1375519

5. Basu, A. and R.W. Blanning, 1992. Metagraphs and petri nets in model management. Proceedings of the Second Annual Workshop on Information Technologies and Systems, Dec. 22-25, Dallas, pp: 64-73.

6. Banerjee, S. and A. Basu, 1993. Model type selection in an integrated DSS environment. Dec. Support Syst., 9: 75-89. DOI: 10.1016/01679236(93)90024-W

7. Barua, A., S.C.H. Lee and A.B. Whinston, 2005. The calculus of reengineering. Inform. Syst. Res., 7: 409-428. DOI: 10.1287/isre.7.4.409

8. Basu, A. and R.W. Blanning, 1992. Enterprise modeling using metagraphs. Proceedings of the IFIP TC8/WG8.3 Working Conference on Decision Support Systems: Experiences and Expectations, June 30-July 3, North-Holland Publishing Co., Amsterdam, The Netherlands, pp: 183-199. http://portal.acm.org/citation.cfm?id=722402

9. Basu, A. and R.W. Blanning, 1997. Synthesis and decomposition of processes in organizations. Inform. Syst. Res., 14: 337-355. DOI: 10.1287/isre.14.4.337.24901

10. Basu, A., R.W. Blanning and A. Shtub, 1997. Metagraphs in hierarchical modeling. Manage. Sci., 43: 623-639. http://portal.acm.org/citation.cfm?id=260571

11. Basu, A. and R.W. Blanning, 1995. Meta-graphs. Omega, 23: 13-25. http://ideas.repec.org/a/eee/jomega/v23y1995i1p13 $-25 . h t m l$

12. Basu, A. and R.W. Blanning, 2005. A formal approach to workflow analysis. Inform. Syst. Res., 11: 17-36. DOI: 10.1287/isre.11.1.17.11787 